\title{
Recall of pure lists of prolonged and repeated (spaced) words
}

\author{
JAMES W. HALL \\ Northwestern University, Evanston, Illinois
}

\begin{abstract}
In a free recall experiment, total study time per item $(2,4$, or $6 \mathrm{sec}$ between subjects) was crossed, with number of item presentations (1 vs. 2 , within subjects). Consequently, each subject studied two lists in which each item was presented once for durations of 2,4 , or $6 \mathrm{sec}$ (the prolonged lists), and two lists in which each item was presented twice, spaced, for durations of 1,2 , or 3 sec, respectively. There was no recall advantage for spaced repetitions in any of the three-study time conditions. For the longer study times, recall from the early serial positions relative to the later positions was greater for the prolonged than for the spaced lists. The results may be accounted for by assuming that displaced rehearsal occurs more during prolonged presentations (or massed repetitions) than during spaced repetitions.
\end{abstract}

Words repeated nonconsecutively (spaced) within a list typically can be recalled better than words repeated consecutively (massed); this phenomenon is commonly known as the spacing effect. An account that has received considerable support (see Hintzman, 1976) posits that this recall difference is the result of study time or attentional difference between spaced and massed repetitions. According to this attenuation of attention hypothesis, the typical subject in some sense "turns off the processor" during massed repetitions, perhaps because of overestimating the learning of the massed items (Zechmeister \& Shaughnessy, 1980).

Another factor that may contribute to the spacing ef fect on free recall is displaced rehearsal. The displacedrehearsal proposal similarly assumes less attention to massed repetitions, but it also assumes that subjects redirect their attention to earlier list items rather than away from the list entirely. The result, according to this hypothesis, is more rehearsal (total study time) for spaced than for massed words within a list. The use of a displaced rehearsal strategy during study for free recall is not at issue; the question is whether such rehearsal favors spaced over massed words. The evidence on this point is equivocal: Waugh (1970, Experiment 2) reported data that fit the account very nicely, whereas Underwood (1969, Experiment 3) found no support for it.

The logic underlying these experimental tests was straightforward. If spacing was manipulated between lists, displaced rehearsal could not benefit spaced more than massed lists, because the total study time for the massed list would equal that for the spaced list. Thus, if displaced rehearsal were the only factor producing the spacing ad-

\footnotetext{
I thank Randy Hove for his help in collecting data. Address correspondence to James W. Hall, Department of Psychology, Northwestern University, Evanston, IL 60208.
}

vantage in the previous mixed-list experiments, there would be no such advantage in the unmixed (betweenlists) situation.

In Underwood's (1969) experiment, there was a recall advantage for spaced lists over massed lists of roughly the same order of magnitude as had been found with mixed lists. This finding suggested that displaced rehearsal played a minor role, at most, in accounting for the spacing advantage found in the earlier mixed-list experiments. Waugh (1970), on the other hand, found no difference in the recall of spaced and massed lists, and internal analyses of her data yielded results that seemed clearly to implicate displaced rehearsal as a factor in producing the spacing advantage in mixed-list spacing experiments. The reason for the conflicting results in the Underwood (1969) and Waugh (1970) experiments and, therefore, the role of displaced rehearsal in producing the spacing effect, remain uncertain, mainly because mixed-list designs continued to dominate spacing experiments. Spacing effects were reported in two additional unmixed-list free recall experiments by Underwood (1970, Experiments 2 and 4), but no mixed-list condition was included in either of those experiments.

In the present experiment, total study time per item (2, 4 , or $6 \mathrm{sec}$, varied between subjects) was crossed with number of item presentations (1 vs. 2, within subjects). This yielded three sets of conditions in which total study time was the same: $1 \times 2 \mathrm{sec}$ versus $2 \times 1 \mathrm{sec}, 1 \times 4 \mathrm{sec}$ versus $2 \times 2 \mathrm{sec}$, and $1 \times 6 \mathrm{sec}$ versus $2 \times 3 \mathrm{sec}$. There were no filler items in the lists. Thus, each subject studied two pure spaced lists, in which every item occurred twice with lags of one or two, and two pure prolonged lists, in which every item occurred once for twice the duration as in the corresponding spaced lists. The displacedrehearsal hypothesis implies that total list recall will be the same for the prolonged and spaced lists for which total study time is the same. Other accounts of the spacing 
effect with mixed lists appear to imply that such an effect will be found with pure lists as well, and with prolonged presentations in place of massed repetitions.

\section{METHOD}

\section{Subjects and Design}

The subjects, 66 Northwestern undergraduates enrolled in the introductory psychology course, were assigned in equal numbers, using random procedures, to one of three total-time conditions: $2 \mathrm{sec}$ per item, $4 \mathrm{sec}$ per item, and $6 \mathrm{sec}$ per item. Within these conditions, subjects were presented two consecutive lists in which every word occurred one time (prolonged lists) and two lists in which every word occurred twice with either one or two other words between repetitions (spaced lists). Half of the 22 subjects in each totaltime condition were given the spaced lists first, and half were given the prolonged lists first.

\section{Materials and Procedure}

Each of the four lists consisted of 20 different five-letter nouns with Thorndike-Lorge frequencies of 20-50 per million, such as fever or slope. Eighty such words were distributed randomly into the four lists, which were given to all subjects in the same order.

Microcomputers were used to present the study instructions, the study lists, a brief serial recall task following the study list, and the test instructions. The study instructions informed the subjects of the list length, the number and rate of presentations per word, and the nature of the interpolated task and the test. The study list was then presented, followed by a set of 6 digits to be memorized and written down. The subjects were then given $2 \mathrm{~min}$ for free recall of the study words. This procedure was followed for each of the four study lists.

\section{RESULTS AND DISCUSSION}

The relevant data are summarized in Table 1 . The most striking feature of these data is the absence of any advantage for the lists of repeated versus prolonged presentations at any of the study-time levels. Indeed, the difference is in the opposite direction.

A 3 (total time per item) $\times 2$ (spaced vs. prolonged, as a repeated measure) $\times 2$ (order of the repeated measure factor) analysis of variance was applied to recall summed over the two lists within each condition. For this and other analyses, the probability of a Type I error was set at .05 .

Only two effects reached statistical significance in this analysis. One was the expected increase in recall as study time increased $\left[F(1,60)=12.67, M S_{e}=70.95\right]$. The second was an uninteresting order effect; recall was greater when the subjects received the prolonged condition first

Table 1

Mean Recall of Prolonged and Repeated (Spaced) Words

\begin{tabular}{llll}
\hline & \multicolumn{3}{c}{ Total Study Time } \\
\cline { 2 - 4 } \multicolumn{1}{c}{ Condition } & $2 \mathrm{sec}$ & $4 \mathrm{sec}$ & $6 \mathrm{sec}$ \\
\hline Prolonged (1 presentation) & 16.68 & 21.95 & 24.59 \\
Repeated (spaced) & 14.64 & 21.14 & 24.50 \\
\hline
\end{tabular}

than it was when the spaced-repetition condition was given first $\left[F(1,60)=9.34, M S_{e}=70.54\right]$. The superiority of the prolonged over the repeated condition was so nearly significant $\left[F(1,60)=3.84, M S_{\mathrm{e}}=8.34, p=.055\right]$ that it seems worth some attention. What is immediately apparent from Table 1 about this difference is that it was due almost entirely to the superiority of the $1 \times 2 \mathrm{sec}$ over the $2 \times 1 \mathrm{sec}$ condition. Perhaps when presentation durations become as brief as $1 \mathrm{sec}$, subjects are unable to reach their maximum levels of effective processing for subsequent recall.

If displaced rehearsal does occur more during massed than during spaced presentations, one might expect a difference to appear in the function relating serial position at input to subsequent recall. Specifically, since early list items tend to receive the bulk of the displaced rehearsal, recall of these items should be relatively higher for prolonged than for spaced lists. Evidence in accord with this expectation was observed when recall of items in the first five and the last five serial positions was examined. For the prolonged lists, the mean recall percentges were 65 for the first five and 51 for the last five positions, whereas the means for the spaced lists were 62 and 56, respectively; the interaction was reliable $[F(1,60)=4.11$, $\left.M S_{\mathrm{e}}=2.69\right]$.

The failure to find any advantage of spaced repetitions seems to eliminate the possibility of any serious attenuation of attention in the present experiment. It does not seem difficult, however, to reconcile this finding with the data reported by Underwood (1969, Experiment 3), because the latter experiment placed much greater demands on attention; there were, in that experiment, longer presentation duratons ( $5 \mathrm{sec}$ per item), more repetitions (as many as 4), and a longer study list (108 positions). It seems likely that the contributions of displaced rehearsal and attenuation of attention to the spacing advantages found in earlier mixed-list free recall experiments varied with the study-time characteristics of the experimental situations.

The results reported here also do not seem to fit other leading theories concerning spacing effects. Specifically, theories that involve either context variability (see, e.g., Glenberg, 1980) or the automatic study-phase retrieval of earlier list items (see, e.g., Hintzman, 1976; Rea \& Modigliani, 1987) imply a spacing advantage with pure lists as well as with mixed lists, and with prolonged presentations as well as massed repetitions. However, those theories are not ruled out as explanations of spacing effects under conditions that do not involve the initiation of a review strategy during study. Displaced rehearsal very likely is not as common a study strategy when subjects expect a recognition or a cued recall test as it is when they anticipate a free recall test. Thus, when a spacing advantage is found in other tasks, in incidental free recall (see, e.g., Jensen \& Freund, 1981) or in free recall by very young children (see, e.g., Rea \& 
Modigliani, 1987), it seems unlikely that displaced rehearsal is heavily involved.

\section{REFERENCES}

GlenberG, A. M. (1979). Component-levels theory of the effects of spacing of repetitions on recall and recognition. Memory \& Cognition, 7, 95-112.

Hintzman, D. L. (1976). Repetition and memory. In G. H. Bower (Ed.), The psychology of learning and memory (Vol. 11, pp. 47-91). New York: Academic Press.

Jensen, T. D., \& FreUnd, J. S. (1981). Persistence of the spacing effect in incidental free recall: The effect of external list comparisons and intertask correlations. Bulletin of the Psychonomic Society, 18, 183-186.
ReA, C. P., Modigliani, V. (1987). The spacing effect in 4- to 9year-old children. Memory \& Cognition, 15, 436-443.

UNDERWOOD, B. J. (1969). Some correlates of item repetition in freerecall learning. Journal of Verbal Learning \& Verbal Behavior, 8, 83-94.

UNDERWOOD, B. J. (1970). A breakdown of the total-time law in freerecall learning. Journal of Verbal Learning \& Verbal Behavior, 9, 573-580.

WAUGH, N. C. (1970). On the effective duration of a repeated word. Journal of Verbal Learning \& Verbal Behavior, 9, 587-595.

Zechmeister, E. B., \& Shaughnessy, J. J. (1980). When you know that you know and when you think that you know but you don't. Bulletin of the Psychonomic Society, 15, 41-44.

(Manuscript received June 17, 1991.) 